\title{
ON THE USE OF CYBER-PHYSICAL HIERARCHY FOR SMART GRID SECURITY AND EFFICIENT CONTROL
}

\author{
Jin Wei, Deepa Kundur and Takis Zourntos \\ Department of Electrical and Computer Engineering \\ Texas A\&M University, College Station, TX 77843, USA
}

\begin{abstract}
We study the application of cyber-physical hierarchy on a class of smart grid systems to improve scalability. Our framework employs a multi-agent flocking-based approach to study the transient stability problem in emerging power systems. An agent in this context embodies a coherent group of system generators. We demonstrate how our paradigm conveniently facilitates the identification of coherent machine clusters through spectral bisection of the associated Kronreduced power system graph. This enables a state-dependent system hierarchy whereby inter-agent interactions are cyber-physical (tier1) and intra-agent synergies are physical (tier-2). By leveraging this layered perspective, active control can be employed only at a select "lead" generator of each agent; secondary generators that are necessarily coherent to a lead generator will naturally follow suit. Thus this cyber-physical hierarchy improves communications and energy overhead by introducing cyber couplings only within components of the smart grid where physical relationships are insufficient for transient stability in the face of a incidental fault or intentional attack. We demonstrate the performance of our approach on the 9-bus WECC system demonstrating its lower overhead and greater robustness to cyber attacks resulting in information delay.
\end{abstract}

Index Terms - cyber-physical system security, smart grid hierarchy, flocking theory.

\section{INTRODUCTION}

The smart grid design mantra for evolution can be described, in part, as "knowledge is power". The application of information technology to the power grid aims to provide more efficient and reliable operation and facilitate new consumer-centric applications through greater situational awareness. Advanced measurement devices and metering provide unprecedented opportunities for informed decision-making. Information no doubt plays an important aspect of enhancing power system operation, but questions naturally arise as to when the increased measurement evokes needless data acquisition. Two practical considerations arise: 1) communications and computational overhead: redundant and irrelevant information acquisition and use will result in excessive computational burden with limited performance return; 2) increasing risks of cyber attack: indiscriminate cyberdependence and -connectivity increases the scope and impact of potential cyber attacks. Thus, in this work, we focus, in part, on understanding how to best harness an appropriate degree of information technology for power system operation.

A preliminary version of this work has been submitted for panel presentation at the 2012 IEEE PES General Meeting. Funding was provided by the U.S. National Science Foundation under grant ECCS-1028246 and the Norman Hackerman Advanced Research Program Project Number 0005120111-2009.
We take a formal cyber-physical perspective and embody cyber and physical interactions of the smart grid through graphical and dynamical systems representations. Cyber elements are introduced only when such interactions enhance the physical goals of the power system. Representing the overall cyber-physical system as a twotier hierarchy enables the effective identification of physical system insufficiencies and corresponding cyber system requirements.

We focus on the problem of transient stability in the presence of a system fault using distributed control based on wide area measurement data. We extend our prior work [1] by introducing hierarchy in the system through identification of coherent generator groups thus providing information on physical couplings that can be leveraged to steer components of the smart grid to a state of stability. Each generator group is embodied by an agent such that inter-agent interactions are cyber-physical in nature. Intra-agent interactions occur amongst coherent generators and are correspondingly physical in nature. The requirements for cyber components and connectivity is reflected by the needs of active control at the agent-level which must drive the overall system to stability.

\section{FLOCKING-BASED FRAMEWORK}

The derivation of our cyber-physical smart grid model is detailed in [1]. Here, we provide a brief overview of our framework. Our construction begins with a smart grid topology and aims to create a scalable depiction relating both physical and cyber components. To address issues of transient stability, these mathematical descriptions must relate generator rotor angles and frequencies. One way to achieve this is to employ the classical swing equation model of a synchronous generator on the Kron-reduced power system. The following well-known equations result for an $N$-generator system:

$$
M_{i} \dot{\omega}_{i}=-D_{i} \omega_{i}+P_{m, i}-\left|E_{i}\right|^{2} G_{i i}-\sum_{j=1}^{N} P_{i j} \sin \left(\theta_{i}-\theta_{j}+\varphi_{i j}\right)
$$

where $i \in\{1,2, \ldots, N\}$ represents the generator index, $\theta_{i}$ denotes the rotor phase angle measured with respect to a rotating frame reference at frequency $f_{0}=60 \mathrm{~Hz}, \omega_{i}=\dot{\theta}_{i}$ is the relative normalized frequency, $M_{i}>0$ and $D_{i}>0$ represent the generator inertia and the damping parameters, respectively, $E_{i}, P_{m, i}$ and $G_{i i}$ are the internal voltage, mechanical power input and equivalent shunt conductance of Generator $i$, respectively. $P_{i j}=\left|E_{i}\right|\left|E_{j}\right|\left|Y_{i j}\right|$ and $\varphi_{i j}=\arctan \left(G_{i j} / B_{i j}\right)$ where $Y_{i j}, G_{i j}$ and $B_{i j}$ are the Kronreduced equivalent admittance, conductance and susceptance, respectively, between Generators $i$ and $j$.

We integrate the influence of the cyber network through an active control strategy in which power injection/absorption is employed at each generator bus to aid in transient stabilization in the face of physical disruption. We assume that a fast-reacting power source $P_{u, i}$ 
facilitated through smart grid technology such as flywheels or battery storage is available and its associated control signal $u_{i}=P_{u, i}$ is computed by employing sensor measurements available through the cyber network. The cyber-physical coupling can therefore be represented as:

$$
\begin{aligned}
M_{i} \dot{\omega}_{i}= & -D_{i} \omega_{i}+P_{m, i}+\underbrace{u_{i}}_{\text {cyber influence }}-\left|E_{i}\right|^{2} G_{i i} \\
& -\sum_{j=1}^{N} P_{i j} \sin \left(\theta_{i}-\theta_{j}+\varphi_{i j}\right) .
\end{aligned}
$$

Eq. (2) carries the flavor of dynamical system models of flocking behavior [2]. Thus, inspired by the analogies present between the requirements for transient stability and flocking rules in nature, we develop a flocking-based control protocol that consists of four terms: a gradient-based term to ensure phase angles of all synchronous generators are within $\frac{5 \pi}{9}$ required for transient stability, a consensus term to enable frequencies of all generators converge, navigation feedback so that frequencies converge to $\omega^{*}$ and a component designed to enable singular perturbation analysis. The resulting cyberphysical system (physical power system with flocking-based cyber control) can be described as [1]:

$$
\left\{\begin{array}{l}
\dot{\boldsymbol{\theta}}=\boldsymbol{\omega} \\
\mathbf{M} \dot{\boldsymbol{\omega}}=\widehat{\mathbf{u}}=-\nabla \mathbf{V}-\mathbf{L} \boldsymbol{\omega}-\mathbf{G} \boldsymbol{\omega}-c_{1}\left(\boldsymbol{\omega}-\boldsymbol{\omega}^{*}\right)
\end{array}\right.
$$

where $\boldsymbol{\theta}=\left[\theta_{1}, \ldots, \theta_{N}\right]^{T}, \boldsymbol{\omega}=\left[\omega_{1}, \ldots, \omega_{N}\right]^{T}, \mathbf{M}=\mathbf{D}(\mathbf{I}+\mathbf{B})$, I is the identity matrix, $\mathbf{D}=\operatorname{diag}\left[D_{1}, \ldots, D_{N}\right], \mathbf{B}$ is a $N \times N$ cyber coupling matrix designed to relax the over-damped generator assumption, $\mathbf{L}$ is the physical coupling matrix which is determined by the impedances of the Kron-reduced power system topology, $\mathbf{G}$ is another $N \times N$ cyber coupling matrix designed to achieve frequency consensus, $\nabla \mathbf{V}$ is the gradient-based term, $c_{1}$ is the parameter for the linear navigational feedback and $\boldsymbol{\omega}^{*}=\mathbf{0}$ is the desired relative normalized generator frequency.

The flocking-based active control strategy requires power injection/absorption at each generator bus to facilitate transient stability and increased critical clearing time. We next describe an approach to leverage the physical coupling amongst generators to apply such control only to "lead" generator buses thus saving control cost while maintaining its functionality.

\section{TWO-TIER HIERARCHICAL CONTROL FRAMEWORK}

Scalability of our flocking-based approach for transient stability is facilitated through exploiting physical couplings amongst generators. We assert that by leveraging generator coherency, active control can be employed only at select lead generators for their stabilization; secondary generators that are necessarily coherent to one or more lead generators will naturally follow suit. To enable such an layered approach requires identifying generator coherency.

\subsection{Spectral Matrix-Based Generator Coherency Identification}

To identify generator coherency, we make use of insights recently presented by Dörfler and Bullo in [3] whereby it is shown via singular perturbation analysis that Eq. (1) is equivalent to:

$$
D_{i} \omega_{i}=P_{m, i}-\left|E_{i}\right|^{2} G_{i i}-\sum_{j=1}^{N} P_{i j} \sin \left(\theta_{i}-\theta_{j}+\varphi_{i j}\right) .
$$

Taking the derivative of both sides, Eq. (4) can be compactly represented as [3]:

$$
\mathbf{D} \dot{\omega}=-\mathbf{L} \omega,
$$

where $\mathbf{D}=\operatorname{diag}\left[D_{1}, D_{2}, \ldots, D_{N}\right], \boldsymbol{\omega}=\left[\omega_{1}, \ldots, \omega_{N}\right]^{T}, \mathbf{L}$ is a $N \times N$ matrix whose elements $l_{i j}$ are defined as:

$$
l_{i j}= \begin{cases}-P_{i j} \cos \left(\theta_{i}-\theta_{j}+\varphi_{i j}\right), & \text { if } i \neq j . \\ \sum_{k=1}^{N-1} P_{i k} \cos \left(\theta_{i}-\theta_{k}+\varphi_{i k}\right), & \text { otherwise. }\end{cases}
$$

It can be shown that the matrix $\mathbf{L}$ can be interpreted as the Laplacian (with zero row-sum and positive semi-definite (PSD) character) of a directed weighted graph $\mathcal{G}$ associated with the power system topology for a small time interval after the fault is cleared; the associated weight of an edge $e_{i j}$ in $\mathcal{G}$ would be given by $l_{i j}$.

It is well known that for Laplacian matrices, the second smallest eigenvalue $\lambda_{2}$ represents the algebraic connectivity of its associated graph. Moreover, for our framework the signs of the elements of the associated eigenvector $\mathbf{v}$ (called the Fedler vector) provide information for spectral bisection $[4,5]$ to partition $\mathcal{G}$ into two relatively disjoint (in terms of physical coupling) subgraphs. In the case of bisection, one group of generators will represent high coherency and the other low.

An algorithm to identify generator coherency is summarized in Table 1. We assume, as typical, that the number of generator groups to partition is known a priori and denoted $\vartheta$; the algorithm is given for $\vartheta$ a power of two, but more general values of coherency groups can be considered if strategies for selective partitioning and joining of subgroups is applied at specific stages. The algorithm is faultdependent and real-time so that it can be applied shortly after a system fault is cleared to analyze coherency just prior to the application of control. After identification of coherent clusters, the two-tier hierarchical controlling framework described in the next section is applied.

\subsection{Two-Tier Hierarchical Control Framework}

In our two-tier hierarchical control framework, the power grid is modeled as a dynamical multi-agent system in which each agent consists of the generators with high physical coupling. The generator groupings within each agent are identified via the spectral matrixbased generator coherency identification algorithm of Table 1 . In each agent, the generator having highest inertia is selected as the lead component and the cyber control is applied only at the lead component. The active control guarantees transient stability of the lead generators (tier-1) and the physical couplings facilitate overall consensus of the secondary generator frequency states (tier-2). As a result all generator frequencies converge to the reference frequency (i.e., typically $60 \mathrm{~Hz}$ ) and phase cohesiveness is established.

Under this tiered framework, the complexity of the overall cyber control protocol is reduced and the cyber control law is established as presented in Eq. 3 and in [1] except that it is applied to the reference graph $\mathcal{G}$ of the two-tier system, created by taking the original graph of the Kron-reduced system and eliminating cyber links of non-lead generators. Similarly, the Laplacian matrix $\widetilde{\mathbf{L}}$ used for control computation is generated by excluding rows and columns from the original system Laplacian $\mathbf{L}$ of Eq. 5 that do not correspond to lead generators.

Intuitively, it is clear that such a tiered approach provides scalability. However, questions naturally arise as to the performance of such an approach in stabilizing the system. What are the compromises with respect to computational complexity, communication 
Table 1. Spectral Matrix Based Generator Coherency Identification Method

Given a predetermined number of the generator groups: $\vartheta=2^{C}$

1. Obtain the Laplacian matrix $\mathbf{L}$ by using Eq. (6).

2. Calculate the eigenvector $\mathbf{v}$ corresponding to the smallest nontrivial eigenvalue of the laplacian matrix.

3. For $k_{1}=1: N$

if the $k$ th element of the eigenvector $\mathbf{v}\left(k_{1}\right)>0$

the $k$ th generator belonging to the partition $\mathcal{S}_{+}$, else

the $k_{1}$ th generator belonging to the partition $\mathcal{S}_{-}$,

End end

4. If $C>1$

for $k_{2}=2: C$

1). Remove the edges connecting the partitions $\mathcal{S}_{+}$and $\mathcal{S}_{-}$from the graph $\mathcal{G}$ and achieve two disconnected graphs $\mathcal{G}_{+}$and $\mathcal{G}_{-}$associated with $\mathcal{S}_{+}$and $\mathcal{S}_{-}$, respectively.

2). For $\mathcal{G}_{+}$and $\mathcal{G}_{-}$, respectively, achieve the associated Laplacian matrix $\mathbf{L}_{+}$and $\mathbf{L}_{-}$and repeat the steps 2 and 3.

end

Else End

and energy overhead? How sensitive is the system to information delay stemming say from denial-of-service cyber attacks? Do noncoherent groupings and the subsequent identification of incorrect lead generators reduce performance? We evaluate the performance of our proposed two-tier hierarchical flocking-based cyber-physical control framework with respect to these metrics for the WECC 9-bus power system.

\section{PERFORMANCE CASE STUDY}

The WECC 9-bus power system power [6] is shown in Fig. 1. In order to demonstrate the utility of the proposed framework for wide area monitoring systems, the normalized impedance of each transmission line is increased from $0.1 j$ (WECC standard) to $0.35 j$. The increase in impedance reduces the physical couplings amongst generators hence making the transient stability problem more challenging.

After Kron-reduction, the dynamic graph associated with the physical system is presented in Fig. 2(a). The graph consists of three nodes representing each of the three generators with associated dynamics described by Eq. (1). The edges represent physical coupling whereby the weight of the edge between $G_{i}$ and $G_{j}$ is given by $Y_{i j}$, the Kron-reduced equivalent admittance between those generators.

Our non-tiered approach to active control would require power injection $P_{u, i}$ at all three generator buses and that all generators exchange information to compute $u_{i}=P_{u, i}$ as shown in Fig. 2(b). Our tiered approach naturally reduces communication overhead as control computation is required only amongst lead generators of each coherence group. For example, Figs. 2(c) and 2(d) represent two possible scenarios depending on the generators' coherence.

For this case study, we assume that a 3-phase short circuit fault occurs at Bus 6 of Fig. 1 at time $t=0 \mathrm{~s}$ and that the associated line is removed at $t=0.3 \mathrm{~s}$, after the critical clearing time. The system

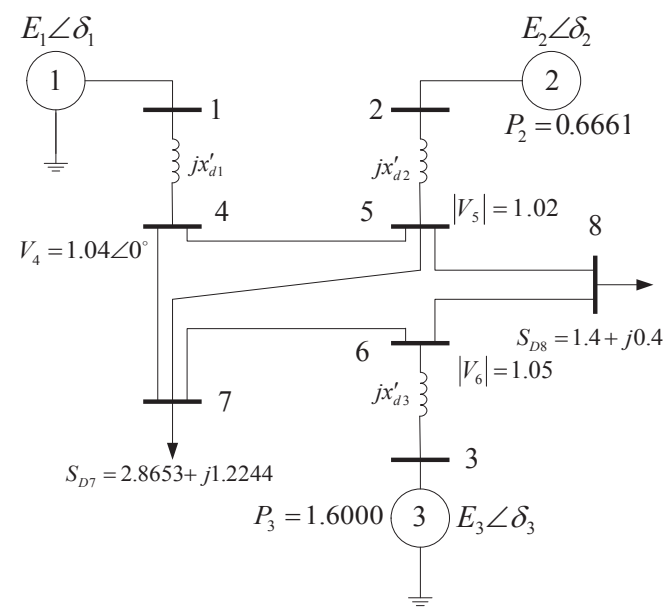

Fig. 1. WECC 9-bus power system [6]; in this work the normalized impedance of each transmission line is increased from $0.1 j$ (WECC standard) to $0.35 j$.

behavior is shown in Fig. 3 over a period of $10 \mathrm{~s}$ and as expected the system does not achieve transient stability; the normalized frequencies and phase angles become unstable and phase angle differences diverge above $5 \pi / 9$. The frequencies and phase angles of $G_{1}$ and $G_{2}$ overlap in plots Figs. 3(a) and (b) and have a zero phase angle difference as observed in Fig. 3(c) forming a coherent group distinct form $G_{3}$.

When the proposed two-tier approach is applied, we assume that the generator coherency is identified at $t=0.4 \mathrm{~s}$, and the control is activated at $t=0.45 \mathrm{~s}$. We identify the generator coherency by using our proposed spectral matrix-based generator coherency identification algorithm. The predetermined number of generator groups is $\vartheta=2$ thus spectral bisection is applied only once. At $t=0.4 \mathrm{~s}$, the Laplacian matrix $\mathbf{L}$, eigenvalue $\lambda_{2}$ and associated Fedler vector $\mathbf{v}$ are given by:

$$
\begin{aligned}
\mathbf{L} & =\left(\begin{array}{ccc}
2.4465 & -1.8037 & -0.6428 \\
-1.8068 & 2.2250 & -0.4182 \\
-0.6184 & -0.3979 & 1.0164
\end{array}\right), \\
\lambda_{2} & =1.5327, \\
\mathbf{v} & =[-0.3663,-0.4715,0.8022]^{T} .
\end{aligned}
$$

Based on the signs of the elements of the eigenvector $\mathbf{v}$, we conclude that the $\vartheta=2$ agents consist of: $\left\{G_{1}, G_{2}\right\}$ and $\left\{G_{3}\right\}$. The accuracy of the coherency identification results is validated by Fig. 3 .

After obtaining generator coherency identification results and selecting the lead component for each agent as the generator with greatest inertia, we achieve the two-tier hierarchical cyber-physical control framework in Fig. 2(c). There are two agents, each consisting of a set of one or more generators with high physical coherency. The cyber links connect the lead generators $G_{1}$ and $G_{3}$ that must compute control signals as a function of one another's physical state. The distributed controllers determine $u_{i}=P_{u, i}$ as detailed in the previous section. A maximum limit on the amount of power injected at each generator bus by the fast reacting grid is assumed; specifically, $P_{u, i} / P_{m, i} \leq 1$ is assumed and for any $u_{i}=P_{u, i}$ computed above this threshold, the power is assumed to be clipped. Fig. 4 presents the normalized system frequency (the reader should note that the desired 


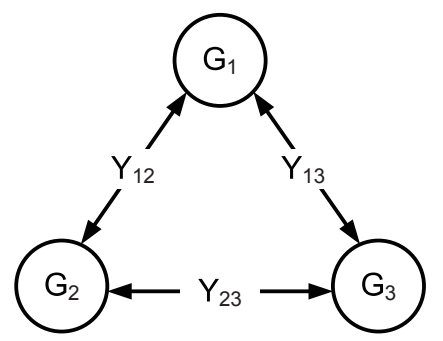

(a)

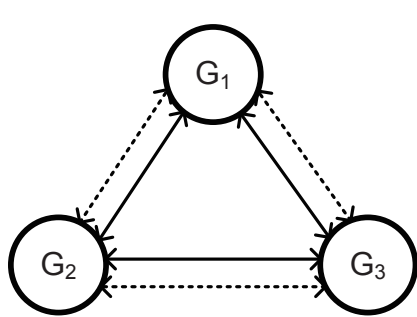

(b)

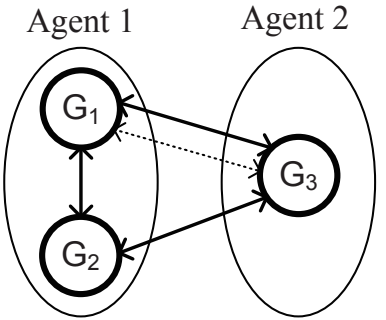

(c)

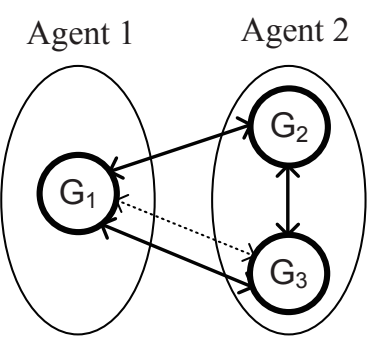

(d)

Fig. 2. WECC 9-bus power system representations. Solid (dashed) lines represent physical (cyber) couplings. (a) Dynamical-graph representation of Kron-reduced system, (b) Cyber-physical couplings without hierarchy, (c) Hierarchical cyber-physical couplings for 3-phase short at Bus 6, (d) Possible two-tier hierarchy.

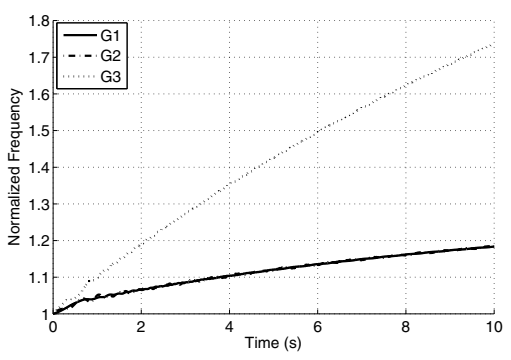

(a) Normalized frequencies versus time.

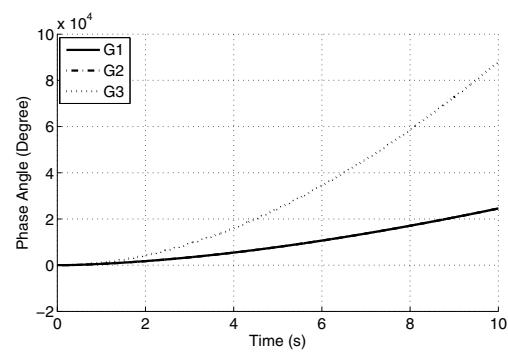

(b) Phase angles versus time.

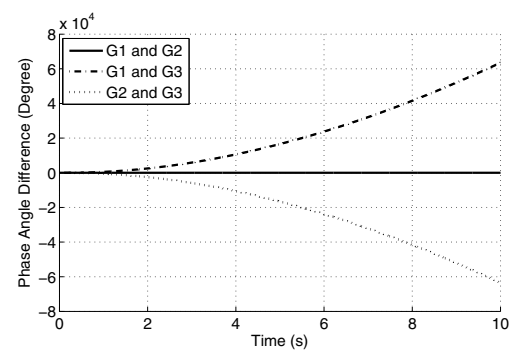

(c) Phase angle differences versus time.

Fig. 3. Results for 3-phase short at Bus 6 when line is removed at $t=0.3 \mathrm{~s}$ without application of flocking-based control; $G_{1}$ and $G_{2}$ form a coherent group distinct from $G_{3}$.

value is unity not zero as for the relative normalized frequency $\omega^{*}$ ) and phase when the two-tier flocking-based cyber-physical control is activated at $t=0.45 \mathrm{~s}$. The injected power is shown in Fig. 6(a) and demonstrates clipping for $G_{1}$ and $G_{3}$; the injected power at bus $G_{2}$ is zero given the tiered architecture and its physical coherence to $G_{1}$.

In contrast, Fig. 2(b) illustrates the flocking-based cyber-physical control framework without the hierarchy [1]. All generators have the same priority and the cyber links exist connecting each generator pair; this full connectivity comes at the cost of communication overhead. Figure 6(b) shows the associated $P_{u, i}$ with clipping as discussed above. Thus, every generator pair is coupled through both cyber and physical means. This is in contrast to Fig. 2(c) in which all generators are coupled physically, but cyber coupling only exists between $G_{1}$ and $G_{3}$. Assuming that the control is activated at $t=0.4 \mathrm{~s}$, Fig. 5 presents the results. Comparing Figs. 4 and 5 we observe that our two-tier framework does not cause noticeable reduction in maintenance of transient stability.

Figure 6(c) compares the total power transmission between the fast-reacting power grid and the WECC 9-bus power system by using the cyber-physical control framework with and without the two-tier hierarchical structure. The area under each power transmission curve determines the total energy exchanged between the fast acting grid and the WECC 9-bus system to enable transient stability in the face of the fault. Thus, the results validate the energy efficiency of our proposed two-tier hierarchical control framework.

In order to evaluate the robustness of our approach to delays in cyber information possibly stemming from denial-of-service cy- ber attacks, we study the performance of our control strategy in the face of information delay. Figs. 7 and 8 evaluate the performances of our hierarchical and non-hierarchical control strategies, respectively, under a communication delay of $\tau=0.05 \mathrm{~s}$; here, the information from neighboring generators used for computation of $u_{i}$ is assumed to be delayed by $\tau$. We observe that our hierarchical structure is more robust to communication delay as transient stability is facilitated whereas for the non-hierarchical case instability results. We believe that the reason for this stems from the greater dependence on physical couplings within the hierarchical approach which makes the approach less susceptible to cyber attacks applied to the information used to compute the control laws.

We emphasize that our two-tier approach requires that generator groupings within agents must exhibit physical coherence for correct identification of lead generators. For example, if for the case of the 3-phase short circuit at Bus 6 the generator groupings do not follow the results of the coherence identification method but instead the lead generators are selected to be $G_{1}$ and $G_{2}$ the hierarchical flockingbased control does not enable transient stability.

\section{FINAL REMARKS}

Overall, we have investigated the application of hierarchy for cyberphysical control and security of smart grid systems. The generators within a smart grid are grouped into coherent clusters such that each cluster is embodied by an agent. The first layer in the two-tier framework reflects cyber-physical interactions amongst lead genera- 


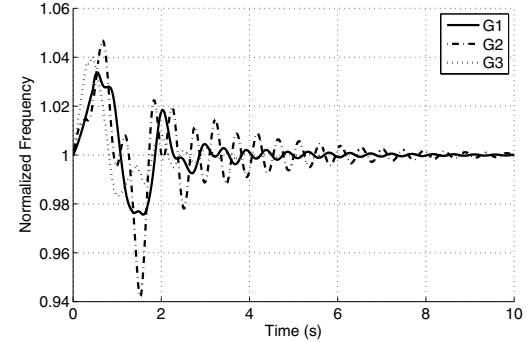

(a) Normalized frequencies.

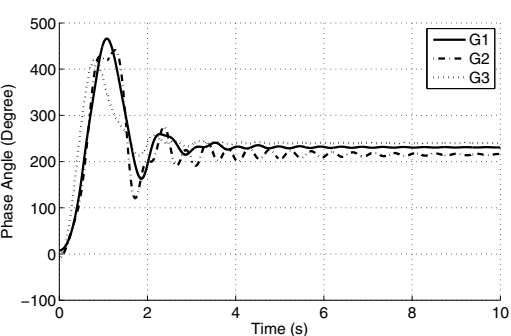

(b) Phase angles.

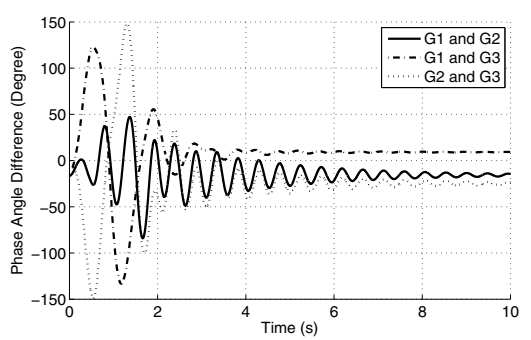

(c) Phase angle differences.

Fig. 4. Results for 3-phase short at Bus 6 when line is removed at $t=0.3 \mathrm{~s}$ with proposed two-tier hierarchical cyber-physical control at $t=0.45 \mathrm{~s}$; no information delay.

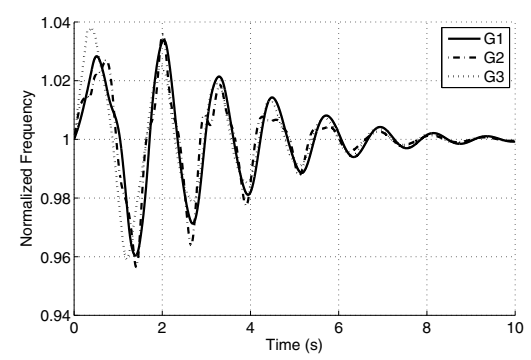

(a) Normalized frequencies.

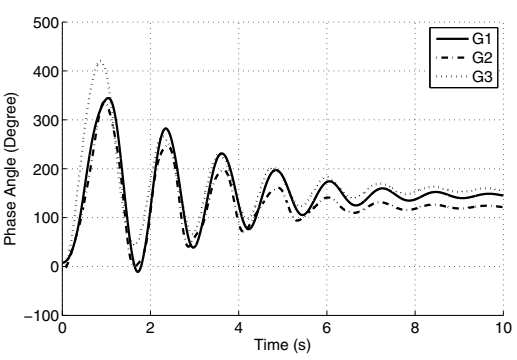

(b) Phase angles.

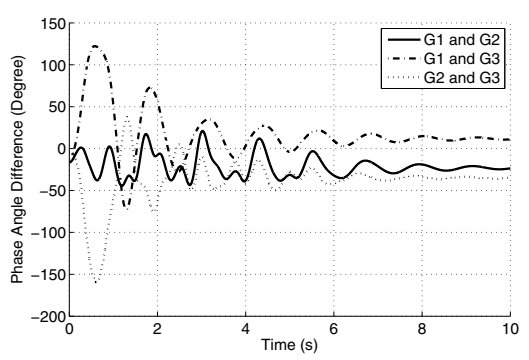

(c) Phase angle differences.

Fig. 5. Results for 3-phase short at Bus 6 when line is removed at $t=0.3 \mathrm{~s}$ with non-hierarchical cyber-physical flocking control at $t=0.4 \mathrm{~s}$; no information delay.

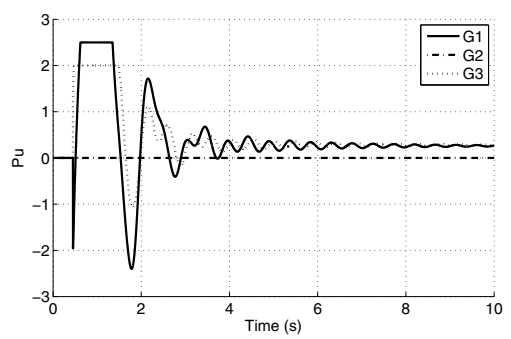

(a) Power transmission $P_{u}$ with hierarchy.

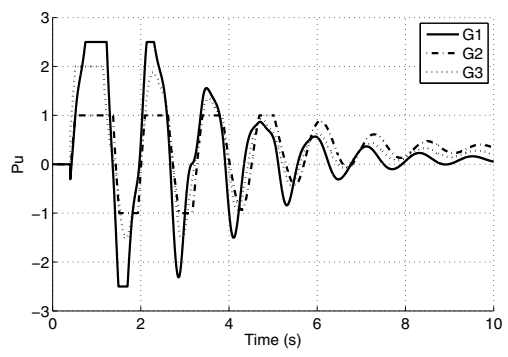

(b) Power transmission $P_{u}$ without hierarchy.

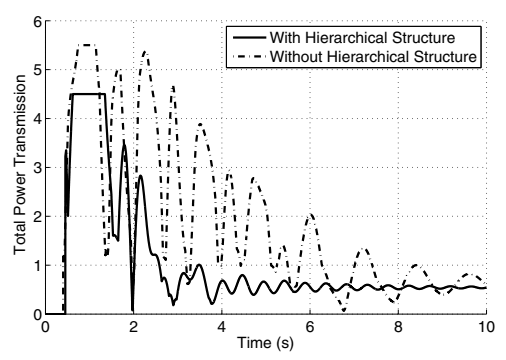

(c) Total power transmission $P_{u}$.

Fig. 6. Power transmission between the fast-reacting power grid and the WECC 9-bus power system for 3-phase short at Bus 6 . 


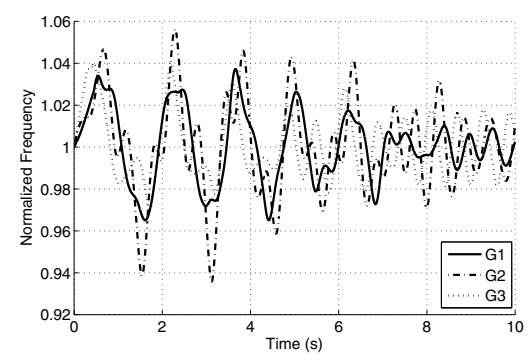

(a) Normalized frequencies versus time.

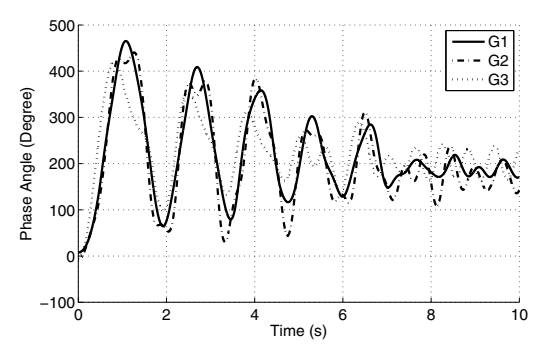

(b) Phase angles versus time.

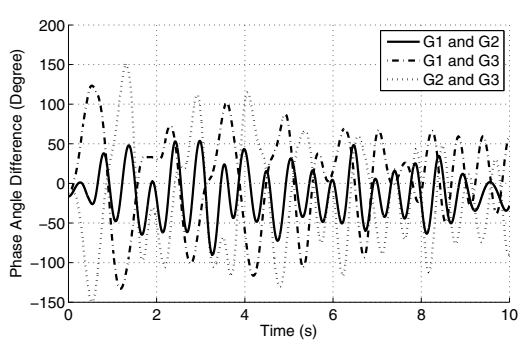

(c) Phase angle differences versus time.

Fig. 7. Results for 3-phase short at Bus 6 when line is removed at $t=0.3 \mathrm{~s}$ with proposed two-tier hierarchical cyber-physical control at $t=0.45 \mathrm{~s} ; \tau=0.05 \mathrm{~s}$.

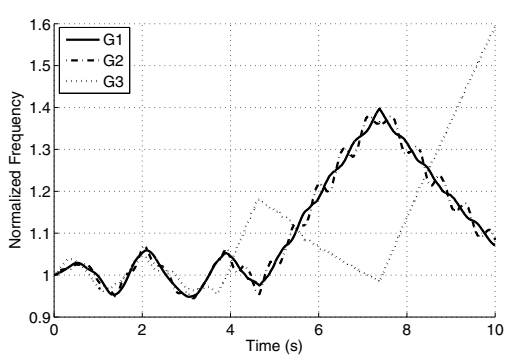

(a) Normalized frequencies versus time.

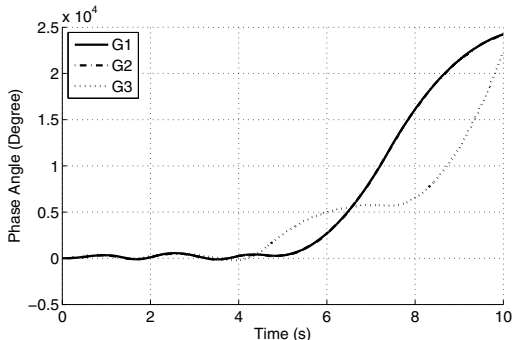

(b) Phase angles versus time.

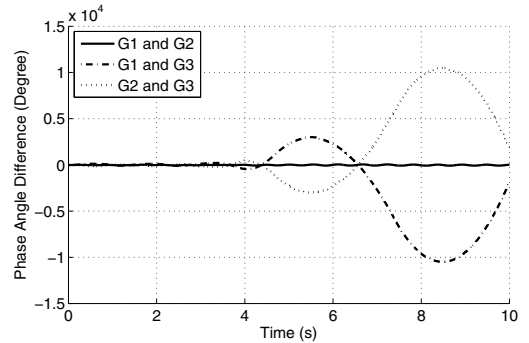

(c) Phase angle differences versus time.

Fig. 8. Results for 3-phase short at Bus 6 when line is removed at $t=0.3 \mathrm{~s}$ with non-hierarchical cyber-physical flocking control at $t=0.4 \mathrm{~s}$; $\tau=0.05 \mathrm{~s}$.

tors of agents and the second layer characterizes physical couplings amongst generators within an agent. Through the study of a cyberphysical flocking-based control protocol on the WECC 9-bus system, we have demonstrated the potential of the approach in reducing communication and energy overhead required for control. This comes at the cost of increased computational complexity required for determining generator coherency for the formation of generatorcluster agents in real-time. However, the hierarchical approach reduces control signal computation and decreases the cyber coupling amongst generators relying, in part, on physical coherence to achieve transient stability in the face of fault or cyber-physical attacks. We observe that this reduced use of information makes the effective cyber-physical system inherently more robust against cyber attacks and information delay.

\section{REFERENCES}

[1] J. Wei, D. Kundur, T. Zourntos, and K. Butler-Purry, "A flocking-based dynamical systems paradigm for smart power system analysis," in Proc. IEEE Power Engineering Society General Meeting, San Diego, CA, July 2012.

[2] R. Olfati-Saber, J. Fax, and R. Murray, "Consensus and cooperation in networked multi-agent systems," Proceedings of the IEEE, vol. 95, no. 1, pp. 215-233, January 2007.

[3] F. Dörfler and F. Bullo, "Topological equivalence of a structurepreserving power network model and a non-uniform Kuramoto model of coupled oscillators," in Proc. IEEE Conference on Decision and Control and European Control Conference, Orlando, FL, December 2011, pp. 7099-7104.

[4] M. Fiedler, "Algebraic connectivity of graphs," Czechoslovak Mathematical Journal, vol. 23, pp. 298-305, 1973.

[5] A. Seary and W. Richards, Dynamic Social Network Modeling and Analysis. National Academies' Press, 2003.

[6] P. Sauer and M. Pai, Power System Dynamics and Stability. Prentice Hall, 1997. 